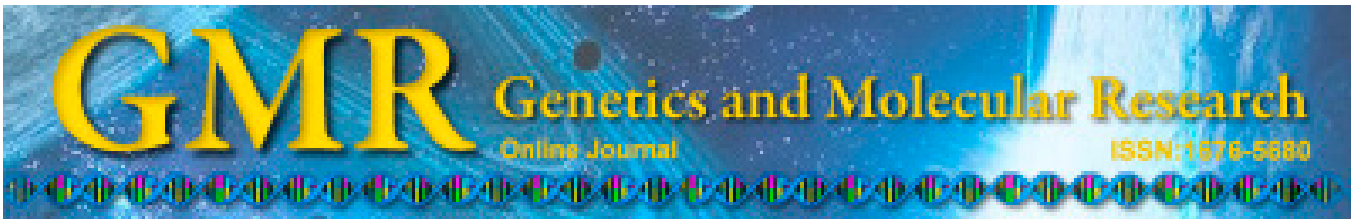

\title{
Adaption of SYBR Green-based reagent kit for real-time PCR quantitation of GC-rich DNA
}

\author{
G.J. Chang ${ }^{1,2}$, H.M. Seyfert ${ }^{2}$ and X.Z. Shen ${ }^{1}$ \\ ${ }^{1}$ College of Veterinary Medicine, Nanjing Agricultural University, \\ Nanjing, China \\ ${ }^{2}$ Leibniz Institute for Farm Animal Biology, Dummerstorf, Germany \\ Corresponding author: X.Z. Shen \\ E-mail: xzshen@njau.edu.cn
}

Genet. Mol. Res. 14 (3): 8509-8515 (2015)

Received December 11, 2014

Accepted April 7, 2015

Published July 28, 2015

DOI http://dx.doi.org/10.4238/2015.July.28.20

\begin{abstract}
In the mammalian genome, approximately $50 \%$ of all genes are controlled by promoters with high GC contents. Analyzing the epigenetic mechanisms regulating their expression is difficult. Hence, we examined a method for stable quantification of such GC-rich DNA sequences. Quantification of DNA during real-time PCR is often based on reagent kits containing the fluorescent dye SYBR Green. However, these ready-made kits may not be suitable for amplifying DNA samples with a high GC content (>70\%). DNA segments with eccentric GC contents are frequently found in proximal promoter areas, and their quantification may be necessary in chromatin accessibility by real-time polymerase chain reaction or chromatin immunoprecipitation analyses of epigenetic mechanisms of gene regulation. We therefore optimized the SYBR Green I FastStart reaction system by supplementing the system with dimethyl sulfoxide, betaine, and increased DNA polymerase content. Here, we describe the development of the assay and demonstrate its effectiveness for two different DNA templates,
\end{abstract}


showing that these modifications allow for the reliable amplification and quantification of DNA with GC contents exceeding $>70 \%$ using the LightCycler instrument.

Key words: Epigenetic mechanisms; GC-rich DNA;

Chromatin accessibility; Real-time polymerase chain reaction

\section{INTRODUCTION}

Polymerase chain reaction (PCR) is a fundamental molecular biology technique that is generally applied in many research fields. However, amplification of GC-rich templates can be difficult (McDowell et al., 1998). Several additives have been described to improve the efficacy of the amplification of GC-rich sequences. These additives include supplementation with dimethyl sulfoxide (DMSO), glycerol, and betaine, either alone or in combination with other components (Henke et al., 1997; Kang et al., 2005; Musso et al., 2006; Mamedov et al., 2008; Li et al., 2011; Pratyush et al., 2012). However, we were unable to determine suitable modifications for commercially available reagent mixes that allowed for reliable quantification of a GC-rich templates using quantitative real-time (qRT)-PCR. DNA segments featuring unusually high GC contents ("GC-islands") occupy only a small percentage (3\%) of the mammalian genome, but approximately $50 \%$ of all genes are controlled by promoters featuring such GCislands (Vavouri and Lehner, 2012). Analyzing the epigenetic mechanisms regulating their expression is difficult because the relevant techniques, such as chromatin immunoprecipitation or chromatin accessibility by real-time PCR (Rao et al., 2001; Vanselow et al., 2006), require reliable determination of the amount of target DNA retrieved.

\section{MATERIAL AND METHODS}

\section{Genomic DNA extraction}

Genomic DNA was extracted from the liver tissue of healthy lactating cows. Briefly, 100 $\mathrm{mg}$ liver tissue was ground into powder under liquid nitrogen, and then $250 \mathrm{mg}$ tissue powder was added to $3 \mathrm{~mL}$ proteinase $\mathrm{K}$ buffer containing $0.5 \mathrm{mg} / \mathrm{mL}$ proteinase $\mathrm{K}$. This solution was mixed gently and incubated at $56^{\circ} \mathrm{C}$ overnight. On the next day, the sample was cooled to room temperature and an equal volume of phenol/chloroform was added, followed by gentle mixing by inverting the tube for $10 \mathrm{~min}$. The tube was centrifuged and the upper aqueous phase was transferred to a new tube. Subsequently, 1/10 volume $3 \mathrm{M}$ sodium acetate, $\mathrm{pH}$ 5.2, was added as well as an equal volume of isopropanol, followed by gentle mixing. The DNA was pelleted by centrifugation and the DNA pellet was washed with $70 \%$ ethanol. Finally, the pellet was air-dried for 10-15 min at room temperature, dissolved in $\mathrm{ddH}_{2} \mathrm{O}$, and stored at $-20^{\circ} \mathrm{C}$.

\section{Retrieval and cloning of high-GC content sequence of candidate gene}

The TLR2 promoter P1A (TLR2-P1A, Gene ID 281534) fragment with a GC content of $78.1 \%$ (Supplementary material) was amplified from genomic DNA using a PCR amplification kit (GC-rich PCR System, REF 12140306001; Roche, Basel, Switzerland) using a touchdown PCR program with the following primers: 5'-ACGTCGGTCAGGAATTAG- 
GAT-3' and 5'-GTCACAGCCGCTTCGAGTGA-3' (forward and reverse, respectively). The amplified target sequence was inserted into the $\mathrm{pGEM}^{\mathbb{B}}-\mathrm{T}$ Easy vector (Promega, Madison, WI, USA) to obtain the standard TLR2-P1A plasmid for absolute quantification in real-time PCR. The correct plasmid sequence was verified by sequencing. The proximal promoter 2 of the acetyl-CoA carboxylase-alpha (ACACA-P2, Gene ID 281590) contains a high GC content (74\%; Supplementary material) and has been previously described (Mao and Seyfert, 2002). Thus, we also cloned the target ACACA-P2 sequence into the pGEM $^{\circledR}$-T Easy vector to further validate our quantification method of the GC-rich DNA fragment.

\section{qRT-PCR for quantifying the GC-rich DNA segment}

The standard plasmids TLR2-P1A and ACACA-P2 were linearized by the enzyme ScaI (Fermentas, Vilnius, Lithuania). The linearized plasmid of TLR2-P1A was used as a template for qRT-PCR to conduct the modified procedures of the commercial SYBR Green I kit. Genomic DNA and linearized plasmid of ACACA-P2 were used to validate the final modified SYBR Green I kit. The primers of both GC-rich sequences for real-time PCR are listed in the Supplementary material. The qRT-PCR products were mixed gently with $2 \mu \mathrm{L}$ loading buffer, and then visualized on a $1.0 \%$ agarose gel using a gel-imagining system.

The SYBR Green I kit (Cat. No. 03515885001) was purchased from Roche. Betaine and DMSO was purchased from Sigma (St. Louis, MO, USA).

\section{RESULTS}

\section{Modification procedures of SYBR Green I kit for amplification of GC-rich segment}

In the present study, the amplification using the original SYBR Green I reaction kit by real-time PCR failed (Figure 1A). Hence, we applied recommendations found in literature regarding suitable additives to PCR reagent kits to allow for amplification of DNA segment with a high GC content. The TLR2-P1A sequence featuring 78\% GC content was used as the template for the modification procedures of the original commercial SYBR Green I kit.

A $10-\mu \mathrm{L}$ assay using the original SYBR Green I reaction system requires preparation of a 5- $\mu \mathrm{L}$ reagent mix consisting of $0.2 \mu \mathrm{L}$ each of $50 \mu \mathrm{M}$ forward and reverse primers, 2.0 $\mu \mathrm{L}$ SYBR Green I master mix, and $2.6 \mu \mathrm{L}$ DEPC water, to which $5 \mu \mathrm{L}$ DNA template solution is added. Our strategy was to keep constant the volumes for primers and the eventually added 5 - $\mu \mathrm{L}$ sample. Keeping the latter volume at $5 \mu \mathrm{L}$ should allow us to maintain sufficient flexibility in different experimental settings. In all optimization experiments, we used $10^{5}$ copies of the linearized plasmid harboring TLR2-P1A as a template. We first modified the original SYBR Green I kit by adding increasing concentrations (2-10\%) of DMSO. The melting temperature of the DNA was inversely related to the DMSO concentration, as expected (Figure $1 \mathrm{~B}$, right panel). The addition of 6,8 or $10 \%$ DMSO eventually yielded sufficient amounts of the appropriate amplicon after 40 amplification cycles. However, the $\mathrm{Ct}$ values were very high (>36). Hence, we added 0.5 or $1 \mathrm{M}$ betaine (stock, $10 \mathrm{M}$ ) to the SYBR Green mixes that had been supplemented with 6,8 , or $10 \%$ DMSO (Figure 1C). Addition of $0.5 \mathrm{M}$ betaine did not effectively improve the $\mathrm{Ct}$ values. However, addition of $1 \mathrm{M}$ betaine to the kit containing $8 \%$ DMSO resulted in efficient amplification of the target and a reasonable melting temperature of 
$89^{\circ} \mathrm{C}$. Mixes containing 6 or $10 \%$ DMSO were both not feasible. The $6 \%$ DMSO supplementation left the melting temperature at $92^{\circ} \mathrm{C}$, while the $10 \%$ DMSO concentration destabilized the DNA to the extent that the acquisition of SYBR Green was too low (Figure 1C, filled green circles).

A

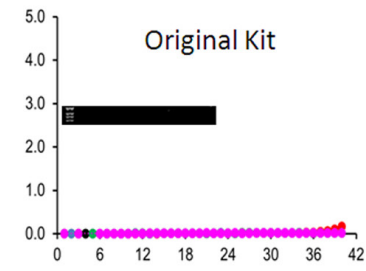

B

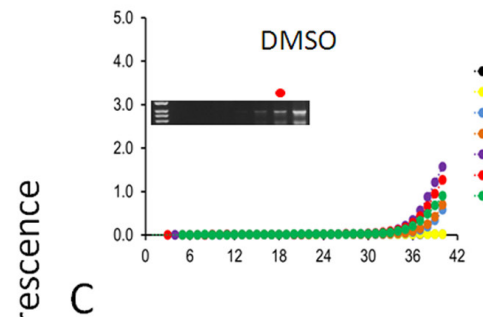

C
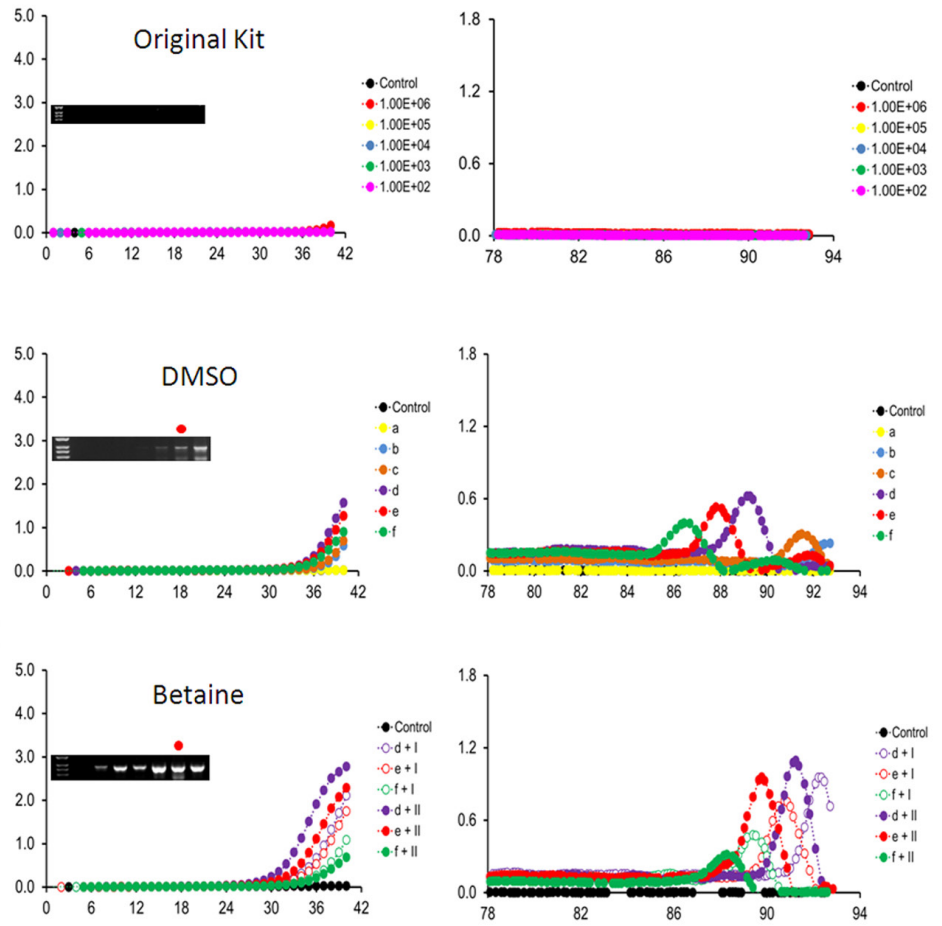

D
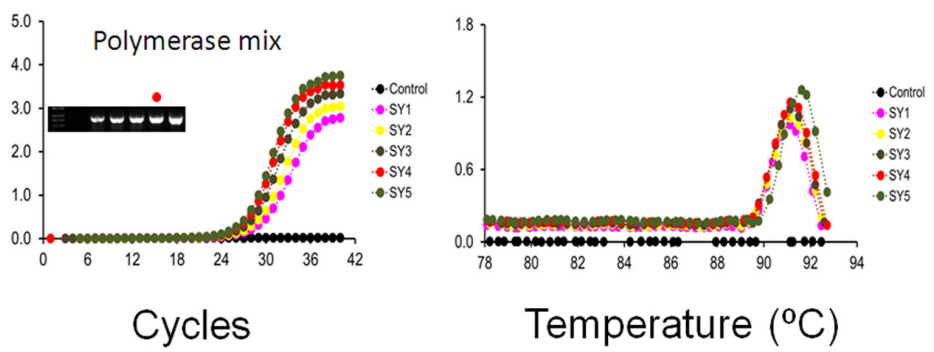

Figure 1. Modification of the original SYBR Green I kit. A. Amplification and melting curve (left hand and right hand panels, respectively) of the linearized TLR2-P1A standard plasmid and the original kit. Different colors of the dotted lines indicate different copy numbers of the standard plasmids added to the assays [black, control (no template added), red to pink, $10^{6}$ to 100 copies added]. B. Modification of original kit (sample a, yellow) with different concentrations of DMSO (2-10\%; b-f, respectively). C. Supplementation of the original kit with a combination of 0.5 or $1.0 \mathrm{M}$ betaine (I and II, respectively) and DMSO $(6,8,10 \%$; d, e, f, respectively). D. Enhancement of the sensitivity of the modified kit ( $8 \%$ DMSO, $1 \mathrm{M}$ betaine) by increasing the concentration of the DNA-polymerase from $2.0 \mu \mathrm{L}$ (SY1, original prescription by stepwise adding $0.2 \mu \mathrm{L}$ polymerase mix (SY2-SY5, respectively). Inserts resolve the PCR products after the LightCycler run on ethidium bromide-stained agarose gels. Red dots above the lanes and red dotted lines specify the results from the respective optimum condition. 
We attempted to further enhance the sensitivity of the assay by increasing the volume added from the SYBR Green master mix from $2.0 \mu \mathrm{L}$ /assay up to $2.8 \mu \mathrm{L}$. This resulted in improved sensitivity (Figure 1D). Adding $2.6 \mu \mathrm{L}$ of this master mix was the best compromise between improved sensitivity and not excessively increasing the melting temperature.

Finally, the master mix of the modified kit contains, per $10 \mu \mathrm{L}$ assay, $2.6 \mu \mathrm{L}$ SYBR Green mix, $0.8 \mu \mathrm{L}$ DMSO, $1.0 \mu \mathrm{L}$ betaine, $0.2 \mu \mathrm{L}$ each of forward and reverse primers, $0.2 \mu \mathrm{L}$ diethylpyrocarbonate-treated water to which $5 \mu \mathrm{L}$ DNA sample may be added. The feasible real-time PCR program using the LightCycler instrument consisted of pre-denaturation at $95^{\circ} \mathrm{C}$ for $10 \mathrm{~min}, 40$ cycles of denaturation at $95^{\circ} \mathrm{C}$ for $30 \mathrm{~s}$, annealing at $60^{\circ} \mathrm{C}$ (primerdependent) for $10 \mathrm{~s}$, elongation at $72^{\circ} \mathrm{C}$ for $500 \mathrm{bp} / \mathrm{min}$, and signal recording at $85^{\circ} \mathrm{C}$ for $5 \mathrm{~s}$.

\section{Validation of the stability of modified kit for quantitation of GC-rich DNA by real-time PCR}

The amplification curve of the TLR2-P1 standard curve using the modified kits illustrated the dependence of amplification kinetics on the concentration of template DNA. We applied $10^{6}-10^{2}$ copies of the linearized plasmid (Figure 2, left upper panel) and found an inverse linear correlation between the log value of the amount of input DNA and the Ct value. Regarding the sensitivity of the assay, we found that 1000 copies of TLR2-P1A can reliably be measured (Figure 2, left middle panel). This is approximately 1/10th of the sensitivity of our standard qRT-PCR assay for non-GC-rich gene templates routinely achieved using this reagent kit (data not shown). The modified assay also reliably quantitates different concentrations of genomic DNA (analyzed 400 to 50 ng; Figure 2, lower left panel).

We validated efficacy of our assay for a different sample of GC-rich DNA. The ACACA-P2 from cattle features also has a high GC-rich content. The analysis of a dilution series of the linearized plasmid containing the target sequence (Figure 2, right upper panel) revealed a clear and significant dose-dependence of the $\mathrm{Ct}$ values based on the amount of template DNA.

\section{DISCUSSION}

Chromatin remodeling underlying the promoter region is a main factor that is highly associated with gene transcription activity (Wiench et al., 2011). We analyzed the chromatin structure underlying the TLR2-P1A region in the liver of cattle using chromatin accessibility by real-time PCR. However, this sequence could only be amplified using the GC-RICH PCR kit with normal PCR, and its amplification using the original SYBR Green I reaction kit by realtime PCR was unsuccessful. Additionally, we could not supplement the GC-rich PCR mix with SYBR Green to allow for DNA quantitation in real-time PCR using the LightCycler Instrument (Roche). Hence, we conducted the present study to develop the current modified SYBR Green I kit and real-time PCR program for reliable amplification and quantitation of GC-rich fragment.

Although numerous advances in the amplification efficiency of real-time PCR have been made in recent years (Rutledge and Stewart, 2008; Chatterjee et al., 2012; Lievens et al., 2012), the amplification of the DNA sequence featuring high-GC contents has not been reported. The kinetic model of quantitative real-time PCR for normal DNA sequences has been well-documented (Gevertz et al., 2005; Mehra and Hu, 2005). Hence, additional studies are needed to elucidate the kinetic mechanism of amplification of GC-rich templates. 
TLR2 P1A

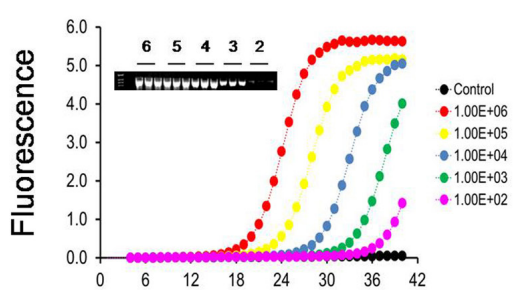

\section{Cycles}
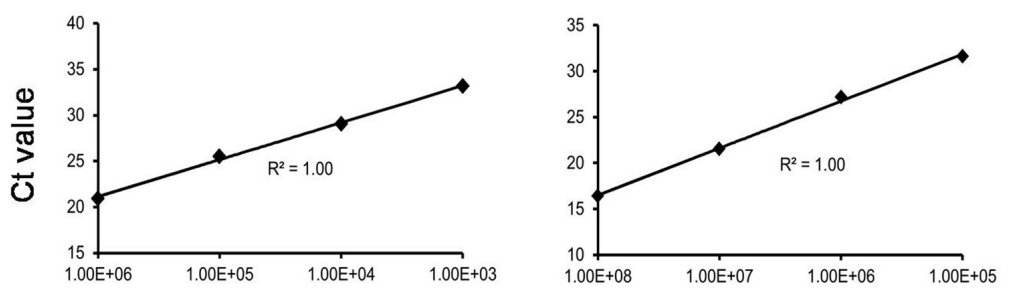

Copy Numbers

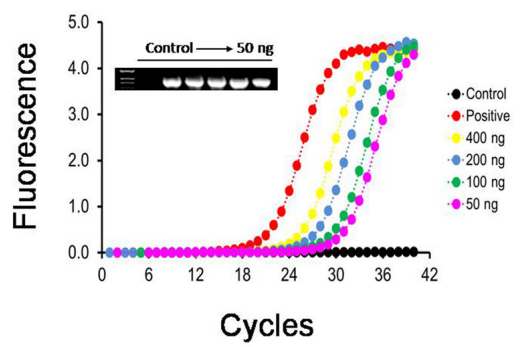

Figure 2. Dose-dependence of the assay. Upper panels: dependence of the amplification kinetics on the concentration of the template DNA; left for TLR2-P1A $\left(10^{6}-10^{2}\right.$ copies); right for ACACA-P2 ( $10^{8}-10^{4}$ copies). Middle panels: respective standard curves. Lower panel: dose-dependence of TLR2-P1A amplification on genomic DNA, positive control (red dotted line) using $10^{6}$ copies of TLR2-P1A standard plasmid.

In summary, our easily applicable modifications of the commercially available SYBR Green I reagent mix allows for reliable quantification of GC-rich DNA sequences. This method can be used for epigenetic techniques, such as chromatin immunoprecipitation or chromatin accessibility by real-time PCR, and is necessary to analyze epigenetic modifications at gene promoters with high GC content $(>70 \%)$.

\section{ACKNOWLEDGMENTS}

Research supported by the National Basic Research Program of China (\#2011CB100802), the National Natural Science Foundation of China (\#31172371), and the Priority Academic Program Development of Jinagsu Higher Education Institutions. 


\section{Supplementary material}

\section{REFERENCES}

Chatterjee N, Banerjee T and Datta S (2012). Accurate estimation of nucleic acids by amplification efficiency dependent PCR. Plos One 7: e42063.

Gevertz JL, Dunn SM and Roth CM (2005). Mathematical model of real-time PCR kinetics. Biotechnol. Bioeng. 92: 346-355.

Henke W, Herdel K, Jung K, Schnorr D, et al. (1997). Betaine improves the PCR amplification of GC-rich DNA sequences. Nucleic Acids Res. 25: 3957-3958.

Kang J, Lee MS and Gorenstein DG (2005). The enhancement of PCR amplification of a random sequence DNA library by DMSO and betaine: application to in vitro combinatorial selection of aptamers. J. Biochem. Biophys. Meth. 64 : 147-151.

Li LY, Li Q, Yu YH, Zhong M, et al. (2011). A primer design strategy for PCR amplification of GC-rich DNA sequences. Clin. Biochem. 44: 692-698.

Lievens A, Van Aelst S, Van den Bulcke M and Goetghebeur E (2012). Enhanced analysis of real-time PCR data by using a variable efficiency model: FPK-PCR. Nucleic Acids Res. 40: e10.

Mamedov TG, Pienaar E, Whitney SE, TerMaat JR, et al. (2008). A fundamental study of the PCR amplification of GCrich DNA templates. Comput. Biol. Chem. 32: 452-457.

Mao JQ and Seyfert HM (2002). Promoter II of the bovine acetyl-coenzyme A carboxylase-alpha-encoding gene is widely expressed and strongly active in different cells. Biochim. Biophys. Acta 1576: 324-329.

McDowell DG, Burns NA and Parkes HC (1998). Localised sequence regions possessing high melting temperatures prevent the amplification of a DNA mimic in competitive PCR. Nucleic Acids Res. 26: 3340-3347.

Mehra S and Hu WS (2005). A kinetic model of quantitative real-time polymerase chain reaction. Biotechnol. Bioeng. 91: 848-860.

Musso M, Bocciardi R, Parodi S, Ravazzolo R, et al. (2006). Betaine, dimethyl sulfoxide, and 7-deaza-dGTP, a powerful mixture for amplification of GC-rich DNA sequences. J. Mol. Diagn. 8: 544-550.

Pratyush DD, Tiwari S, Kumar A and Singh SK (2012). A new approach to touch down method using betaine as co-solvent for increased specificity and intensity of GCrich gene amplification. Gene 497: 269-272.

Rao SH, Procko E and Shannon MF (2001). Chromatin remodeling, measured by a novel real-time polymerase chain reaction assay, across the proximal promoter region of the IL-2 gene. J. Immunol. 167: 4494-4503.

Rutledge RG and Stewart D (2008). Critical evaluation of methods used to determine amplification efficiency refutes the exponential character of real-time PCR. BMC Mol. Biol. 9: 96.

Vanselow J, Yang W, Herrmann J, Zerbe H, et al. (2006). DNA-remethylation around a STAT5-binding enhancer in the alpha S1-casein promoter is associated with abrupt shutdown of alpha S1-casein synthesis during acute mastitis. $J$. Mol. Endocrinol. 37: 463-477.

Vavouri T and Lehner B (2012). Human genes with CpG island promoters have a distinct transcription-associated chromatin organization. Genome Biol. 13: R110.

Wiench M, Miranda TB and Hager GL (2011). Control of nuclear receptor function by local chromatin structure. FEBS J. 278: 2211-2230. 